\title{
ON THE LIFTING OF BOUNDED SETS IN FRÉCHET SPACES
}

\author{
by JOSÉ BONET and SUSANNE DIEROLF
}

(Received 31st May 1991)

\begin{abstract}
This paper considers the behaviour of a quotient map between Fréchet spaces concerning the lifting of bounded sets. The main result shows that a quotient map between Fréchet spaces that lifts bounded sets with closure (or equivalently such that its strong transpose is a topological isomorphism) must also lift bounded sets without closure.
\end{abstract}

1991 Mathematics subject classification: 46A04

In the last few years, the behaviour of a quotient map with respect to bounded sets (i.e. lifting of bounded sets, lifting with closure, and no lifting at all) has occurred more and more frequently in the context of structural investigations and examples of Fréchet and (DF) spaces. Our main result shows that a quotient map between Fréchet spaces that lifts bounded sets with closure also lifts bounded sets.

Let $E$ be a locally convex space, let $F \hookrightarrow E$ be a linear subspace, and let $q: E \rightarrow E / F$ denote the quotient map. We say that $q$ lifts bounded sets if for every bounded subset $B \subset E / F$ there is a bounded subset $A \subset E$ such that $q(A) \supset B$. The classical KötheGrothendieck example of a Fréchet Montel space $E$ admitting $l^{1}$ as a quotient shows that in general even for $E, F$ Fréchet the quotient map $q: E \rightarrow E / F$ may not lift bounded sets. (cf. [12].) On the other hand, we obtain from the Banach-Dieudonne theorem a positive statement: If $E$ is Fréchet and $F$ is closed in $E$ such that $E / F$ is a Montel space, then $q: E \rightarrow E / F$ lifts bounded sets.

Moreover, by a classical result of Palamodov, Merzon, and De Wilde (see [8]), a Fréchet space $F$ is quasinormable if and only if for every Fréchet space $E$ containing $F$ as a topological subspace, the quotient map $q: E \rightarrow E / F$ lifts bounded sets. Finally, we have the following result of Cholodovskij [7]: If $E$ is a quasinormable Frechet space and $F \hookrightarrow E$ is a closed linear subspace such that $q: E \rightarrow E / F$ lifts bounded sets, then $F$ is also quasinormable.

Given again the situation $q: E \rightarrow E / F$ with $E$ locally convex, we have the following well known equivalence: the inclusion $q^{t}:\left(F^{0}, \beta\left(F^{0}, E / F\right)\right) \rightarrow\left(E^{\prime}, \beta\left(E^{\prime}, E\right)\right)$ is a topological isomorphism onto its range if and only if $q: E \rightarrow E / F$ lifts bounded sets with closure, i.e. for every bounded subset $B$ of $E / F$ there is a bounded subset $A$ in $E$ such that $\overline{q(A)} \supset B$.

For the Fréchet Montel space $E$ admitting $l^{1}$ as quotient, the corresponding quotient map $q: E \rightarrow E / F \cong l^{1}$ does not even lift bounded sets with closure. Embedding $E$ topologically into a countable product $G=\prod_{n \in N} G_{n}$ of Banach spaces, one obtains a 
quotient map $G \rightarrow G / F$ which does not lift bounded sets with closure, where both $G$ and $G / F$ are isomorphic to quotients of countable products of Banach spaces.

The concept of "lifting bounded sets with closure" has played an important rôle for certain structural questions in the context of Fréchet spaces:

Let $0 \rightarrow F \rightarrow E \stackrel{q}{\rightarrow} E / F \rightarrow 0$ be a short exact sequence of Frechet spaces. Assume that $q$ lifts bounded sets with closure. If $F$ and $E / F$ are both distinguished (resp. both satisfy the Stefan Heinrich's density condition), then also $E$ is distinguished (resp. satisfies the density condition). (See [5, Proposition 1].) The hypothesis on $q$ is essential.

Let $E$ by a Fréchet space, and let $q: E^{\prime \prime} \rightarrow E^{\prime \prime} / E$ denote the quotient map. If $E^{\prime \prime}$ is distinguished, then $q$ lifts bounded sets with closure and $E^{\prime \prime} / E$ is distinguished. (See [6, Lemma 1].) On the other hand if $E$ and $E^{\prime \prime} / E$ are both distinguished and if $q$ lifts bounded sets with closure, then also $E^{\prime \prime}$ is distinguished.

This last result was an essential help in order to find a distinguished Fréchet space whose bidual is not distinguished. The first examples were given in [6]. Examples in the context of Köthe echelon spaces of order infinity later appeared in Bastin [1].

In $[11$, p. $260 \mathrm{ff}$.] we find a positive result in another context: Given a df-space $E$ and a closed subspace $F \hookrightarrow E$, then the quotient map $q: E \rightarrow E / F$ will always lift bounded sets with closure. For the definition and properties of df-spaces we refer to [11]. Every DFspace of Grothendieck, and hence the strong dual of every Fréchet space and every (LB)-space, is a df-space.

For a (DF)-space $E$ and a closed subspace $F \hookrightarrow E$ it may happen that $q: E \rightarrow E / F$ lifts bounded sets with closure but does not lift bounded sets without closure, as the following well-known example shows. Let $G=$ ind $G_{n}$ be any LB-space. The addition map q: $\oplus_{n \in N} G_{n} \rightarrow G, q\left(\left(x_{n}\right)\right):=\sum_{n \in N} x_{n}$ always lifts bounded sets with closure since $\bigoplus_{n \in N} G_{n}$ is a DF-space. On the other hand $q$ lifts bounded sets if and only if $G$ is a regular LB-space. Now, non-regular LB-spaces abound. See e.g. [12, 31.6], [2] or [3].

The next example will point out that for a Fréchet space $E$ and a closed subspace $F$ in $E$ it may happen that there exist a bounded set in $E / F$ which is not "liftable with closure" as well as a set which is "liftable with closure" but non-"liftable". In this example $F$ will be a distinguished Fréchet space and we may even obtain both $E$ and $E / F$ to be countable products of Banach spaces.

Example 1. Let $X, Y$ be Banach spaces with unit balls $A$ and $B$, respectively, such that $Y \subset X, B \subset A$ and such that $Y$ is not closed in $X$.

Then the map $q: c_{0}(X) \times Y^{\mathrm{N}} \rightarrow c_{0}(X)+Y^{\mathrm{N}}$, with $q\left(\left(x_{n}\right)_{n \in \mathbb{N}},\left(y_{n}\right)_{n \in N}\right):=\left(x_{n}+y_{n}\right)_{n \in N}$ is a quotient map (where $c_{0}(X)+Y^{\mathrm{N}}$ as an algebraic subspace of $X^{\mathrm{N}}$ is provided with the quotient topology). The sets $\varepsilon A^{\mathbb{N}} \cap c_{0}(X)+\prod_{k<n}\{0\} \times \prod_{k \geqq n} Y(\varepsilon>0, n \in \mathbb{N})$ form a 0 -basis in $c_{0}(X)+Y^{N}$.

The space $F:=\operatorname{ker} q$ is topologically isomorphic to the Fréchet space of Moscatelli type w.r. to $Y \hookrightarrow X$ and $c_{0}$ via the isomorphism $\left(z_{n}\right)_{n \in N} \mapsto\left(\left(z_{n}\right)_{n \in N},\left(-z_{n}\right)_{n \in N}\right)$ and therefore $a$ distinguished Fréchet space by $[4,2.5]$.

The space $E:=c_{0}(X) \times Y^{N}$ is a countable product of Banach spaces and therefore a quojection. If we choose $Y$ to be dense in $X$, we get that $c_{0}(X)+Y^{N}=X^{N}$ algebraically, whence, by the open mapping theorem, also $c_{0}(X)+Y^{N}=X^{N}$ holds topologically. 
We define $\mathfrak{B}_{l}:=\{B \subset E / F: \exists A \subset E$ bounded s.t. $B \subset q(A)\}, \mathfrak{B}_{l}:=\{B \subset E / F: \exists A \subset E$ bounded s.t. $B \subset \overline{q(A)}\}, \mathfrak{B}:=\{B \subset E / F: B$ bounded $\}$ and claim that the following inclusions are strict:

$$
\mathfrak{B}_{l} \subset \mathfrak{B}_{I} \subset \mathfrak{B} .
$$

Proof. We first note that a fundamental system in $\mathfrak{B}_{l}$ is given by the scalar multiples of the sets $A^{N} \cap c_{0}(X)+\prod_{k \in N} \delta_{k} B\left(\left(\delta_{k}\right)_{k \in N} \in \omega\right)$.

For every such $\left(\delta_{k}\right)_{k \in N}$ we have

$$
\begin{aligned}
\overline{A^{\mathrm{N}} \cap c_{0}(X)+\prod_{k \in N} \delta_{k} B} & \subset \prod_{k \in N} \overline{A+\delta_{k} B^{X}} \cap\left(c_{0}(X)+Y^{N}\right) \subset 2 \prod_{k \in N}\left(A+\delta_{k} B\right) \cap\left(c_{0}(X)+Y^{\mathrm{N}}\right) \\
& \subset 2 \overline{A^{\mathrm{N}} \cap c_{0}(X)+\prod_{k \in N} \delta_{k} B} .
\end{aligned}
$$

We must only prove the last inclusion. Let $z=\left(z_{k}\right)_{k \in N} \in \prod_{k \in N}\left(A+\delta_{k} B\right) \cap\left(c_{0}(X)+Y^{N}\right)$, $\varepsilon>0, n \in \mathbb{N}$ be given. There are $x=\left(x_{k}\right)_{k \in N} \in c_{0}(X), y=\left(y_{k}\right)_{k \in N} \in Y^{N}$ such that $z=x+y$. Moreover, there is $m \geqq n$ such that $x_{k} \in \varepsilon A$ for all $k \geqq m$, whence $z=\left(\left(z_{k}\right)_{k<m},(0)_{k \geqq m}\right)+$ $\left((0)_{k<m},\left(x_{k}\right)_{k \geqq m}\right)+\left((0)_{k<m},\left(y_{k}\right)_{k \geqq m}\right) \in\left(A^{\mathrm{N}} \cap c_{0}(X)+\prod \delta_{k} B\right)+\varepsilon A^{\mathrm{N}} \cap c_{0}(Y)+\prod_{k<n}\{0\} \times \prod_{k \geqq n} Y$.

Consequently, a fundamental system in $\mathfrak{B}_{\bar{l}}$ is given by the scalar multiples of the sets $\prod_{k \in N}\left(A+\delta_{k} B\right) \cap\left(c_{0}(X)+Y^{N}\right)$ with $\left(\delta_{k}\right)_{k \in N} \in \omega$.

In order to show that the inclusion $\mathfrak{B}_{l} \subset \mathfrak{B}_{\bar{l}}$ is strict, we will prove that $(A \cap Y)^{N}$, which belongs to $\mathfrak{B}_{\bar{l}}$, does not belong to $\mathfrak{B}_{l}$. In fact, let $\left(\delta_{k}\right)_{k \in N}$ be an arbitrary sequence of positive reals. Since $Y$ is not closed in $X$, we obtain from [4, 2.8 and 2.9]:

$$
\exists \varepsilon>0 \forall m \in \mathbb{N} A \cap Y \not \subset \varepsilon A+m B .
$$

We choose $a_{k} \in(A \cap Y) \backslash\left(\varepsilon A+\delta_{k} B\right)(k \in \mathbb{N})$ and obtain that $a:=\left(a_{k}\right)_{k \in N} \in(A \cap Y)^{N}$ does not belong to $c_{0}(X)+\prod \delta_{k} B$, since every $x=\left(x_{k}\right)_{k \in N} \in c_{0}(X)$ lies eventually in $\varepsilon A$.

Utilizing the next theorem we could already conclude that the strict inclusion of $\mathfrak{B}_{\bar{l}}$ in $\mathfrak{B}$ is also satisfied. Nevertheless we will explicitly show that $\prod_{k \in N}(k A \cap Y) \in \mathfrak{B} \backslash \mathfrak{B}_{\Gamma}$. In fact, from the shape of the 0 -neighbourhoods in $c_{0}(X)+Y^{N}$ we easily obtain that $\prod_{k \in N}(k A \cap Y)$ is bounded in $c_{0}(X)+Y^{N}$.

Assume there are $\delta,\left(\delta_{k}\right)_{k \in N}$ positive such that $\prod_{k \in N}(k A \cap Y) \subset \prod_{k \in N}\left(\delta A+\delta_{k} B\right)$. Dividing the $k$ th component by $k(k \in \mathbb{N})$ we get $\prod_{k \in N}(A \cap Y) \subset c_{0}(X)+\prod_{k \in N}\left(\delta_{k} / k\right) B$ which is not true as was shown above.

The Fréchet Montel space with $l^{1}$ as a quotient shows that also $\mathfrak{B}_{\bar{l}}=\mathfrak{B}_{l}$ and $\mathfrak{B}_{l}$ strictly included in $\mathfrak{B}$ may occur, and of course also $\mathfrak{B}=\mathfrak{B}_{l}=\mathfrak{B}_{l}$ can happen. The fourth and last combinatorial possibility in this setting is equivalent to the natural question whether "lifting with closure" will imply "lifting without closure" in the context of Fréchet spaces. The next theorem gives a positive answer to that question:

Theorem 2. Let $E$ be a Fréchet space and let $F \subset E$ be a closed linear subspace such that the quotient map q: $E \rightarrow E / F$ lifts bounded sets with closure, then $q$ lifts bounded sets. 
Proof. Let $B_{0} \subset E / F$ be bounded. Then there is a bounded and absolutely convex subset $A_{1} \subset E$ such that $\overline{q\left(A_{1}\right)} \supset B_{0}$. Since $E / F$ is metrizable, we can apply a classical result of Grothendieck $([10]$ or $[13,5.1 .27])$ to obtain a bounded and absolutely convex subset $B_{1} \subset E / F, B_{1} \supset \overline{q\left(A_{1}\right)}$, such that the spaces $\left(\left[B_{1}\right], p_{B_{1}}\right)$ and $E / F$ induce the same topology on $\overline{q\left(A_{1}\right)}$, hence $\overline{q\left(A_{1}\right)}=\bigcap_{\varepsilon>0}\left(q\left(A_{1}\right)+\varepsilon B_{1}\right)$.

Proceeding by induction, we find sequences $\left(A_{n}\right)_{n \in N}$ of bounded and absolutely convex subsets of $E$ and $\left(B_{n}\right)_{n \in N}$ of bounded and absolutely convex subsets of $E / F$ such that

$$
B_{n-1} \subset \overline{q\left(A_{n}\right)} \subset \bigcap_{\varepsilon>0}\left(q\left(A_{n}\right)+\varepsilon B_{n}\right) \subset B_{n}(n \in \mathbb{N}) .
$$

Since $E$ is metrizable there is a sequence $\left(\alpha_{n}\right)_{n \in N}$ of positive reals such that $C:=\overline{\Gamma\left(\bigcup_{n \in N} \alpha_{n} A_{n}\right)}$ is bounded in $E$ and such that $B:=\overline{\Gamma\left(\bigcup_{n \in N} \alpha_{n+1} B_{n}\right)}$ is bounded in $E / F$. We may and will assume that $\alpha_{1}=2$. Then also $A:=\bigcup_{k \in N} \sum_{n=1}^{k}\left(1 / 2^{n}\right) \alpha_{n} A_{n}$ is a bounded subset in $E$, and we will prove that $q(A) \supset B_{0}$.

Let $b_{0} \in B_{0}$ be given. Because of $b_{0} \in\left(\alpha_{1} / 2\right) q\left(A_{1}\right) \subset q\left(\left(\alpha_{1} / 2\right) A_{1}\right)+\left(1 / 2^{2}\right) \alpha_{2} B_{1}$ there are $a_{1} \in\left(\alpha_{1} / 2\right) A_{1}$ and $b_{1} \in\left(1 / 2^{2}\right) \alpha_{2} B_{1}$ such that $b_{0}=q\left(a_{1}\right)+b_{1}$. Since

$$
\frac{1}{2^{n+1}} \alpha_{n+1} B_{n} \subset \frac{1}{2^{n+1}} \alpha_{n+1} \overline{q\left(A_{n+1}\right)} \subset q\left(\frac{1}{2^{n+1}} \alpha_{n+1} A_{n+1}\right)+\frac{1}{2^{n+2}} \alpha_{n+2} B_{n+1}
$$

for all $n \in \mathbb{N}$, we may proceed by induction and obtain sequences $\left(a_{k}\right)_{k \in N} \in \prod_{k \in N}\left(1 / 2^{k}\right) \alpha_{k} A_{k},\left(b_{k}\right)_{k \in N} \in \prod_{k \in N}\left(1 / 2^{k+1}\right) \alpha_{k+1} B_{k}$ such that $b_{0}=q\left(\sum_{k=1}^{n} \alpha_{k}\right)+b_{n}$ for all $n \in \mathbb{N}$.

Since $b_{k} \in\left(1 / 2^{k+1}\right) B(k \in \mathbb{N})$, the sequence $\left(b_{k}\right)_{k \in N}$ converges to 0 in $E / F$.

Furthermore, since $\sum_{k=n}^{m} a_{k} \in\left(1 / 2^{n-1}\right) C$ for all $m \geqq n$, the sequence $\left(\sum_{k=1}^{n} a_{k}\right)_{n \in N}$ is a Cauchy sequence in the normed space $\left([C], p_{C}\right)$. Since $C$ is a closed absolutely convex bounded subset of the Fréchet space $E$, the normed space $\left([C], p_{C}\right)$ is complete and there is a $a \in[C]$ with $a=\lim _{n \rightarrow \infty} \sum_{k=1}^{n} a_{k}$ in $\left([C], p_{C}\right)$ and consequently in $E$. Clearly $a \in A$ and

$$
q(a)=\lim _{n \rightarrow \infty} q\left(\sum_{k=1}^{n} a_{k}\right)=\lim _{n \rightarrow \infty} \sum_{k=1}^{n} q\left(a_{k}\right)+\lim _{n \rightarrow \infty} b_{n}=\lim _{n \rightarrow \infty}\left(\sum_{k=1}^{n} q\left(a_{k}\right)+b_{n}\right)=b_{0}
$$

which completes the proof.

A. Peris proved and pointed out to us that Theorem 2 remains true if $E$ is metrizable and $F$ is a complete subspace of $E$.

Our main result has already been applied in infinite holomorphy by Díaz [9].

Corollary 3. Let $E$ be a Fréchet space, let $F \hookrightarrow E$ be a closed linear subspace and let $q: E \rightarrow E / F$ denote the quotient map. The following are equivalent:

(i) $\left(F^{0}, \beta\left(E^{\prime}, E\right) \cap F^{0}\right)$ is an LB-space;

(ii) $\left(F^{0}, \beta\left(E^{\prime}, E\right) \cap F^{0}\right)$ is bornological; 
(iii) $\left(F^{0}, \beta\left(E^{\prime}, E\right) \cap F^{0}\right)$ is barrelled;

(iv) $E / F$ is distinguished and $q$ lifts bounded sets.

Proof. (i) $\rightarrow$ (ii) $\rightarrow$ (iii) are immediate.

(iii) $\rightarrow$ (iv): Since $\beta\left(F^{0}, E / F\right) \supset \beta\left(E^{\prime}, E\right) \cap F^{0} \supset \sigma\left(F^{0}, E / F\right)$, we obtain from (iii) that $\beta\left(F^{0}, E / F\right)=\beta\left(E^{\prime}, E\right) \cap F^{0}$ which implies that $\beta\left(F^{0}, E / F\right)$ is barrelled and $q$ lifts bounded sets with closure, hence without closure by the theorem.

(iv) $\rightarrow$ (i) Since $q$ lifts bounded sets, we have $\beta\left(F^{0}, E / F\right)=\beta\left(E^{\prime}, E\right) \cap F^{0}$ and since $E / F$ is distinguished we get that $\left(F^{0}, \beta\left(F^{0}, E / F\right)\right)$ is an LB-space.

Acknowledgement. The research of J. Bonet was partially supported by DGICYT Proyecto $\mathrm{n}^{\circ}$ PS88-0050. The authors thank the referee for his remarks and suggestions which improved the paper and removed an error in the first version.

\section{REFERENCES}

1. F. Bastin, Distinguishedness of weighted Fréchet spaces of continuous functions, Proc. Edinburgh Math. Soc. (2) 35 (1992), 271-283.

2. K. D. Bierstedt, R. G. Meise and W. H. Summers, Köthe sets and Köthe sequence spaces, in Funct. Anal., Holomorphy and Approx. Theory (North-Holland Math. Studies 71, Amsterdam 1982), 27-91.

3. J. Bonet and S. Dierolf, On LB-spaces of Moscatelli type, Dog̃a Mat. 13 (1989), 9-33.

4. J. Bonet and S. Dierolf, Fréchet spaces of Moscatelli type, Rev. Mat. Univ. Complut. Madrid 2 (1989), 77-92.

5. J. Bonet, S. Dierolf and C. Fernandez, On the three-space-problem for distinguished Fréchet spaces, Bull. Soc. Roy. Sci. Liège 59 (1990), 301-306.

6. J. Bonet, S. Dierolf and C. Fernández, The bidual of a distinguished Fréchet space need not be distinguished, Archiv Math. 57 (1991), 475-478.

7. V.E. Cholodovsku, On quasinormability of semimetrizable topological vector spaces, Funkc. Anal. 7 (1976), 157-160.

8. M. DE WILDE, Sur le relèvement des parties bornées d'un quotient d'espaces vectoriels topologiques, Bull. Soc. Roy. Sci. Liège 5-6 (1974), 299-301.

9. J. C. Diaz, A note on holomorphic functions of bounded type in Fréchet spaces, J. Math. Anal. Appl., to appear.

10. A. Grothendieck, Topological Vector Spaces (Gordon and Breach, New York 1973).

11. H. Jarchow, Locally Convex Spaces (B.G. Teubner, Stuttgart 1981).

12. G. Köthe, Topological Vector Spaces I, II (Springer-Verlag, Heidelberg 1969, 1979).

13. P. Perez Carreras and J. Bonet, Barrelled Locally Convex Spaces (North-Holland Math. Studies 131, Amsterdam 1987).

Universidad Politecnica de Valencia

Departamento de matematica Aplicada

E.T.S. Arquitectura

E-46071 VALENCIA

Spain
FB Mathematik IV UNIVERSITÄT TRIER

Postfach 3825

D-5500 Trier

Germany 